\title{
Microbiota of Foregut and Hindgut of Blunt Snout Bream (Megalobrama amblycephala)
}

\author{
Liangkun Pan', Qun-Lan Zhou' ${ }^{1 *}$, Bo Liu ${ }^{1}$, Jiaxing Yan² and Ajun Sun ${ }^{1}$ \\ ${ }^{1}$ Key Laboratory of Freshwater Fisheries and Germplasm Resources Utilization, \\ Ministry of Agriculture, Freshwater Fisheries Research Center, Chinese Academy of \\ Fishery Sciences, Wuxi 214081, China \\ ${ }^{2}$ College of Information Science and Technology, Nanjing Agricultural University, \\ Nanjing, Jiangsu, China
}

\begin{abstract}
A B S T R A C T
To explore the gut microbial profile of blunt snout bream (Megalobrama amblycephala), fish were fed with a commercial diet for 16 weeks. Then high-throughput sequencing was applied to compare the microbiota between foregut and hindgut. The results showed that the microbial profiles between the foregut and hindgut were different based on the alpha-diversity and the cluster results. Dominant microbioorganisms of blunt snout bream at genus level were Cetobacterium spp., Lactococcus spp., CK-1C4-19, Rhodobacter spp. and Pseudomonas spp. Meanwhile it was found out that Lactococcus spp. preferred the foregut, while Cetobacterium spp. and Flavobacterium spp. preferred the hindgut. Dominant bacteria preferred different gut section may decided by its function and physiological characteristics.
\end{abstract}
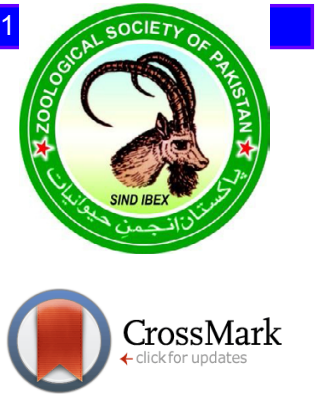

\begin{tabular}{l} 
Article Information \\
\hline Received 18 October 2019 \\
Revised 11 January 2020 \\
Accepted 04 March 2020 \\
Available online 26 October 2020 \\
Authors' Contribution \\
Q-LZ conceived and designed the \\
study, helped in preparation of \\
manuscript. LP wrote the article. BL \\
helped in preparation of manuscript. \\
JY analyzed the data. AS helped in \\
samples collection and rearing of fish. \\
Key words \\
\hline Gut microbiota, Blunt snout bream, \\
High-throughput sequencing, \\
Cetobacterium spp., Lactococcus spp.
\end{tabular}

\section{INTRODUCTION}

$\mathrm{G}^{\mathrm{n}}$ ut microbiota has attracted increased attention in the past ten years after the use of 454 pyrotags to analyze the microbial profile began. It is accepted that the large and diverse bacterial community is very important to nutrient digestion and absorption, and to the health of the gut and the immunity of the host (Jiang et al., 2011; Desai et al., 2012; Wu et al., 2013; Ingerslev et al., 2014; Ye et al., 2014; Zhang et al., 2017). It has also been confirmed that microorganisms in the gut can improve the health of host gut cells by synthesizing important nutrients, such as short-chain fatty acids, peptide, amino acid etc. (Borsodi et al., 2017; Hao et al., 2017). It was well known that fish species (Li et al., 2012; 2014) and the daily diet (He et al., 2013; Ingerslev et al., 2014; Li et al., 2015) could be affected the composition and function of microbiota in the gut of fish (Reveco et al., 2014).

The gut microbiota of the freshwater fish species such as grass carp (Ctenopharyngodon idella) was analyzed (Jiang et al., 2011; Wu et al., 2012), and then more studies of gut microbiota were conducted on gibel carp (Carassius auratus gibelio) (Wu et al., 2013; Li et al., 2017), bighead carp (Aristichthys nobilis) (Li et al., 2014) and black carp (Mylopharyngodon piceus) (He et al., 2013). There are a few reports on the gut microbiota of blunt snout bream

\footnotetext{
* Corresponding author: zhouql@ffrc.cn 0030-9923/2021/0001-0001 \$ 9.00/0

Copyright 2021 Zoological Society of Pakistan
}

(Megalobrama amblycephala) (Li et al., 2012; He et al., 2013). Moreover, there are few reports on the microbial community differences between different gut parts of the fish. Therefore, the aim of this study was to explore the microbial profile of blunt snout bream and to compare the microbiota between foregut and hindgut.

\section{MATERIALS AND METHODS}

\section{Rearing system}

The trail was conducted in the cylindrical fiber glass tanks (300 L water per tank) that were part of a recirculating system, equipped with a sedimentation tank that contained activated carbon and corallite stones as a biological filter. All tanks were equipped with aeration. Water temperature ranged from 27 to $29^{\circ} \mathrm{C}$, which was controlled by a water temperature control system. $\mathrm{pH}$ was 7.4-7.8 and dissolved oxygen was approximately $6 \mathrm{mg} \mathrm{L}^{-1}$ throughout the trial.

\section{Fish and feeding}

A total of 150 juvenile blunt snout breams (initial weight $1.31 \pm 0.45 \mathrm{~g}$ ) were divided into 5 tanks with 30 fish in each tank, which were provided by the fish farm of Freshwater Fisheries Research Center, Chinese Academy of Fishery Sciences in China. Fish were fed with the commercial diet (330 $\mathrm{g} \mathrm{Kg}^{-1}$ crude protein, $15.82 \mathrm{MJ} \mathrm{Kg}^{-1}$ ) (Tongwei Co., Ltd. Wuxi, China), which was formulated using the ingredients such as fishmeal, soybean meal, canola meal, cottonseed meal, wheat flour, rice bran etc. The feed trail lasted for 16 weeks. Fish were hand-fed four 
times a day at 8:00, 10:30, 13:30 and 16:00 until apparent satiation on the basis of visual observation. Pellets were distributed slowly, permitting all fish to eat. The final body weight of the fish was varied from $9.66 \mathrm{~g}$ to $14.67 \mathrm{~g}$, with average final weight $12.02 \pm 1.64 \mathrm{~g}$.

\section{Sample collection}

After 16 weeks, fish were fasted for $24 \mathrm{~h}$ before collecting samples. Six fish from each tank were sampled and anaesthetized by MS-222 (tricainemethanesulfonate, $100 \mathrm{mg} \mathrm{L}^{-1}$, Sigma, USA) and then dissected to obtain the whole gut without digesta. After the complete intestine was removed from the fish, the contents of the intestine were scraped off with a spatula to retain the slimy intestinal sample. The gut was divided into two sections based on the length, the anterior third of the intestine was considered as the foregut and the remainder was considered as the hindgut. Individual gut samples were placed in Eppendorf tubes, immediately put into liquid nitrogen and stored at $-80{ }^{\circ} \mathrm{C}$ until DNA extraction.

Thus, we got 5 foregut samples and 5 hindgut samples, that was S1F (foregut of sample 1), S1H (hindgut of sample 1), S2F (foregut of sample 2), S2H (hindgut of sample 2), $\mathrm{S} 3 \mathrm{~F}$ (foregut of sample 3), $\mathrm{S} 3 \mathrm{H}$ (hindgut of sample 3), S4F (foregut of sample 4), S4H (hindgut of sample 4) and S5F (foregut of sample 5), $\mathrm{S} 5 \mathrm{H}$ (hindgut of sample 5).

\section{DNA extraction, PCR amplification and pyrosequencing}

DNA was isolated from the gut content samples using QIAamp Fast DNA Stool Mini Kit (QIAGEN, Gemany), according to the manufacturer's instructions. DNA concentration was detected using Nanodrop (Thermo scientific, USA). 6 DNA samples from six different fish of the same tank obtained from the same section were mixed in equimolar amount to make a pooled sample.

For the 454 pyrosequencing, an amplicon library was prepared using eubacterial universal primers. Primers with special barcodes were used to amplify $450 \mathrm{bps}$ of the 16S rRNA genes covering the V3 to V4 regions. Primers were as follows: 343F 5'-TACGGRAGGCAGCAG-3', 798R 5'-AGGGTATCTAATCCT-3'. PCR was carried out using polymerase (Trans Start Fastpfu DNA Polymerase, Transgen Biotech, Beijing, China) with an annealing temperature of $52^{\circ} \mathrm{C}$ and 30 cycles to minimize PCR biases. Purified PCR products were submitted for pyrosequencing as described in the PE300 protocol using Illumina Miseq (Illumina, USA) in the Shanghai Majorbio Co. Ltd (Shanghai, China).

Sequence processing, OUT assignment, identification and classification

Sequences generated by pyrosequencing were filtered to remove the ambiguous sequences, homologous sequences and short length sequences from the resulting raw data set, provided by Illumina Miseq, using Trimmomatic software (Bolger et al., 2014), then edited using FLASH software (Reyon et al., 2012). Operational Taxonomic Units (OTUs, the equivalent of species) were picked using a closed-reference protocol at $97 \%$ similarity, as described by CD-HIT software (Li and Godzik, 2006). Reads that did not match any reference sequence with at least $97 \%$ identity were discarded. OTUs were classified and assigned taxonomic identities based on their best match with database Greengenes and Genebank of NCBI using the softwares RDP classifier and PyNAST.

\section{Statistical analyses}

The relative abundance of micro community was assessed at phylum and genus level were calculated based on the OUT number using software QIIME. The dominant microorganism was identified based on its relative abundance among all other microorganisms. Alpha diversity indices were determined using the ShannonWiener index, Simpson index for diversity and the Chao1 index for species richness, which were calculated using software QIIME. $P$ value were calculated using SPSS 19.0 T test. A heatmap with cluster at genus level was draw based on the abundance of gut microbiota using the software Fast Tree. The dominant microbiota was selected based on the relative abundance. A boxplot was drawn based on the relative abundance of dominant microbiota using software SPSS 19.0, to compare the different distributions between the foregut and hindgut.

\section{RESULTS}

Based on the alpha-diversity analysis, the Chao 1 index, Shannon Wiener index and Simpson index were all higher in the hindgut than the foregut, but the difference was not significant.

Table I. Alpha diversity on foregut and hindgut microbiota of blunt snout bream.

\begin{tabular}{llll}
\hline Group & Foregut & Hindgut & P value \\
\hline Chao1 $^{\mathrm{a}}$ & $669.40 \pm 51.08$ & $684.80 \pm 72.86$ & 0.87 \\
Shannon $^{\mathrm{b}}$ & $4.40 \pm 0.49$ & $4.61 \pm 0.29$ & 0.72 \\
Simpson $^{\mathrm{b}}$ & $0.82 \pm 0.06$ & $0.90 \pm 0.02$ & 0.28 \\
\hline
\end{tabular}

Note: ${ }^{a}$ Chao1, estimated OTU richness; ${ }^{b}$ diversity index (Shannon and Simpson). Data was shown as mean \pm STDE. $P$ value was obtained from T-test of SPSS (version 19.0). * means the significant difference when $P<0.05$.

The dominant microbiota at the phylum level of blunt snout bream were Proteobacteria (30.1\%), Tenericutes 
(20.8\%), Fusobacteria (18.4\%), Firmicutes (15.9\%) and Bacteroidetes $(10.3 \%)$. The dominant microbiota at genus level included Cetobacterium (18.3\%), Lactococcus (14.0\%), CK-1C4-19 (9.1\%), Rhodobacter (8.14\%) and Pseudomonas (7.34\%). Based on the cluster of all samples, they could be clustered into two broad categories of foregut and hindgut samples except $\mathrm{S} 1 \mathrm{H}$, which was clustered with foregut.

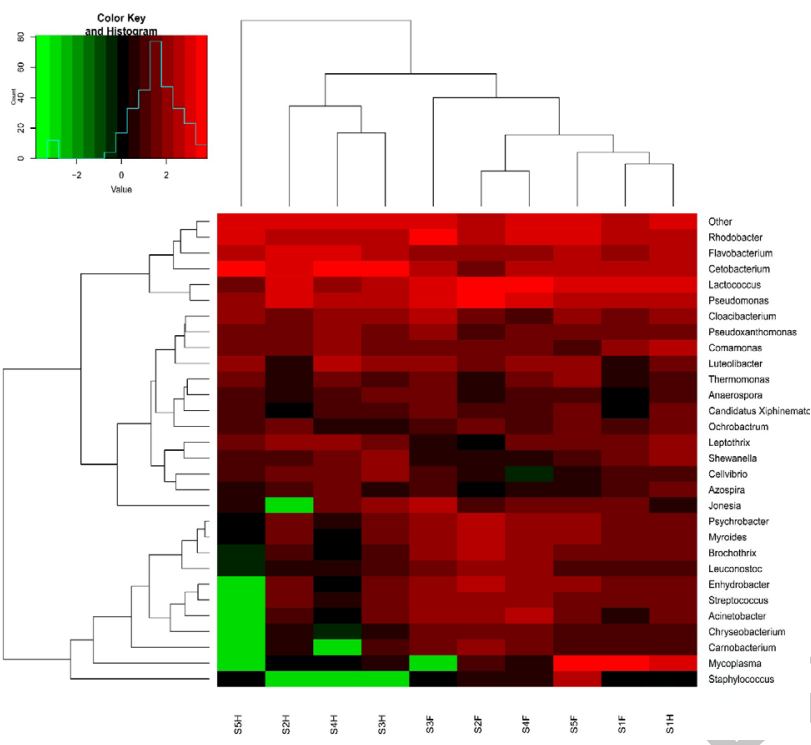

Fig. 1. Relative abundance (\%) of the dominant taxa present in the foregut and hindgut of the blunt snout bream at genus taxonomic level with cluster.

The dominant phylum was different between the foregut and hindgut, which was Firmicutes (25.88\%) in the foregut and Fusobacteria $(33.52 \%)$ in the hindgut. As shown in Table II and Figure 2, the percentage of core microbiota at the genus level varied greatly between the foregut and the hindgut. Lactococcus $(22.62 \%)$ preferred the foregut, while Cetobacterium (33.42\%), CK-1C4-19 $(14.52 \%)$ and Flavobacterium $(7.82 \%)$ were usually found in the hindgut.

\section{DISCUSSION}

The alpha-diversity was higher in the hindgut than the foregut. That means not only the microbiota diversity but also the species richness was high in the hindgut than in the foregut. This may be attributed to the different functions of foregut and hindgut and the differences in the chemical environment of these two sections (Sullam et al., 2012). Meanwhile it is well known that high diversity of the microbiome could make this system more stable, this may be the reason why the alpha-diversity between the foregut and hindgut was not significant. The cluster results were showed that the microbiota of foregut and hindgut were different too. This may mean we should choose the part of gut to study based on the research purpose.

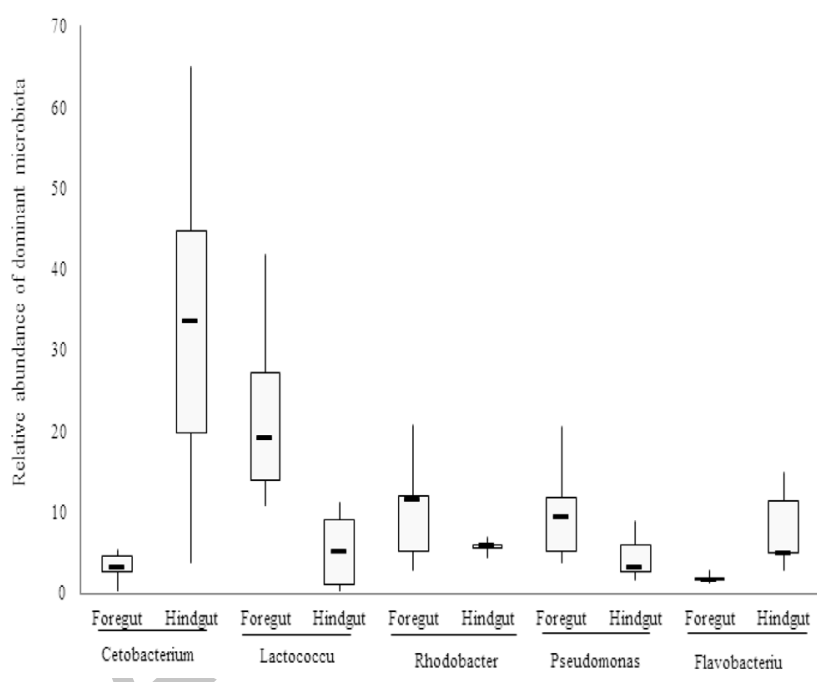

Fig. 2. Box plot of dominant microbiota at the genus level in foregut and hindgut of blunt snout bream.

Table II. Dominant gut microbiota in foregut and hindgut of blunt snout bream at phylum and genus level.

\begin{tabular}{llll}
\hline & Foregut & Hindgut & P value \\
\hline Phylum & & & \\
Proteobacteria & $34.36 \pm 5.67$ & $25.82 \pm 4.21$ & 0.26 \\
Tenericutes & $23.82 \pm 12.18$ & $17.7 \pm 4.86$ & 0.65 \\
Fusobacteria* & $3.32 \pm 0.87$ & $33.52 \pm 10.48$ & 0.02 \\
Firmicutes* & $25.88 \pm 6.17$ & $5.84 \pm 2.28$ & 0.02 \\
Bacteroidetes & $7.2 \pm 1.68$ & $13.44 \pm 2.45$ & 0.07 \\
Genus & & & \\
Cetobacterium* & $3.24 \pm 0.86$ & $33.42 \pm 10.47$ & 0.02 \\
Lactococcus* & $22.62 \pm 5.55$ & $5.38 \pm 2.14$ & 0.02 \\
CK-1C4-19* & $3.7 \pm 2.33$ & $14.52 \pm 2.68$ & 0.02 \\
Rhodobacter & $10.46 \pm 3.12$ & $5.82 \pm 0.40$ & 0.18 \\
Pseudomonas & $10.18 \pm 2.95$ & $4.5 \pm 1.30$ & 0.12 \\
Flavobacterium* & $1.84 \pm 0.27$ & $7.82 \pm 2.29$ & 0.03 \\
\hline
\end{tabular}

Note: Data was shown as mean \pm STDE. P value was obtained from T-test of SPSS (version 19.0). * means the significant difference when $P<0.05$.

The dominant microbiota at the phylum level of blunt snout bream was similar to previous studies carried out on the larvae of blunt snout from a commercial fish farm (Li et al., 2012) and on the blunt snout bream from Wuhu Lake (Li et al., 2014). However, performing the analysis 
at genus level, results were different. Only Cetobacterium was considered as one of the dominant genera of blunt snout bream in the previous study ( $\mathrm{Li}$ et al., 2015). Cetobacterium was also the dominant genera in several other fish species, including the herbivorous grass carp, the filter-feeding big head carp, omnivorous common carp (Cyprinus carpio), Japanese white crucian carp (Carassius cuvieri), bluegill (Lepomis macrochirus), the carnivorous channel catfish (Ictalurus punctatus) and largemouth bass (Micropterus salmoides) (van Kessel et al., 2011; Roeselers et al., 2011; Larsen et al., 2014; Li et al., 2015; Hao et al., 2017). Lactoccus was one of the dominant bacterial genera found in the grass carp too (Li et al., 2015). Similar to the report on grass carp, Pseudomonas was another genus of core microbiota found in blunt snout bream (Li et al., 2015). Previous studies have indicated that Pseudomonas is an important biological control agent in aquaculture that can degrade certain toxic compounds and remove harmful residual materials from fish intestines (Nam et al., 2003).

Based on the cluster result, microbiota in the foregut and hindgut were divided into two groups. But the microbiota in the hindgut of Sample 1 was clustered with foregut group. This might because the dominant genus of Cetobacterium in the $\mathrm{S} 1 \mathrm{H}$ was as low as that in the foregut. On the other hand, it suggested that gut microbes vary greatly between individuals.

It was found in this study that Lactococcus, Rhodobacter and Pseudomonas preferred the foregut, while Cetobacterium and Flavobacterium usually found in the hindgut. Lactococcus is a notable probiotic bacteria, which can not only help the host to maintain a healthy gut environment by producing antibacterial substances that inhibit the spread of harmful intestinal bacteria and suppress growth of competing bacteria (Dawood et al., 2016), but it can also ferment $\alpha$-galactosides, such as melibiose and raffinose (Boucher et al., 2003). Meanwhile, it preferred the foregut maybe decided by the chemical environment of gut, for it is a facultative anaerobe.

Cetobacterium was considered to relate to protein digestion, for it could ferment peptone to produce acetic and propionic acids (Tsuchiya et al., 2008). Acetic and propionic acids are short chain fatty acids that can improve mucosal morphology, preserve enterocyte ultrastructure, and positively impact the host's health via prevention of certain diseases (Hao et al., 2017). It was preferred the hindgut, which was in agreement with the report on grass carp (Hao et al., 2017). The preference of Cetobacterium to the hindgut can be attributed to its function in fermenting peptone to produce acetic and propionic acids (Tsuchiya et al., 2008).

In conclusion, this study provides an insight into the gut microbiota of the blunt snout bream. The results reveal that the diversity of the foregut and hindgut were similar, but the microbiota was different. Some dominant bacteria preferred to plant in different sections of gut, which decided by its function and physiological characteristics. More studies are still needed to identify the function of certain dominant genera in freshwater fish such as Cetobacterium and Lactococus.

\section{ACKNOWLEDGEMENT}

This work was financially supported by Central Public-interest Scientific Institution Basal Research Fund, Freshwater Fisheries Research Center, CAFS, Grant/ Award Number: 2017JBFM16 and 2015JBFM19; the Natural Science Foundation of Jiangsu Province, Grant/ Award Number: BK20170219; the National Natural Science Foundation of China, Grant/Award Number: 31772867; the China Agriculture Research System special project of the National Conventional Freshwater Fishery Industry, Grant/Award Number: CARS-46.

\section{Statement of conflict of interest}

The authors have declared no conflict of interest.

\section{Ethical approval}

The scientific research protocols of the Chinese Academy of Fishery Sciences (CAFS) and the Ministry of Agriculture, PR China, and all relevant local and/ or international animal welfare laws, guidelines and policies for the care and use of animals were followed by the authors.

\section{REFERENCES}

Bolger, A.M., Lohse, M. and Usadel, B., 2014. Trimmomatic: a flexible trimmer for Illumina sequence data. Bioinformatics, 30: 2114-2120. https://doi.org/10.1093/bioinformatics/btu170

Borsodi, K.A., Szabó, A., Krett, G., Felföldi, T., Specziár, A. and Boros, G., 2017. Gut content microbiota of introduced bigheaded carps (Hypophthalmichthys spp.) inhabiting the largest shallow lake in Central Europe. Microbiol. Res., 195: 40-50. https://doi. org/10.1016/j.micres.2016.11.001

Boucher, I., Vadeboncoeur, C. and Moineau, S., 2003. Characterization of genes involved in the metabolism of $\alpha$-galactosides by Lactococcus raffinolactis. Appl. environ. Microbiol., 69: 40494056. https://doi.org/10.1128/AEM.69.7.40494056.2003

Dawood, M.A.O., Koshio, S., Ishikawa, M., Yokoyama, S., Basuini, M.F.E., Hossain, M.S., Nhu, T.H., 
Dossou, S. and Moss, S.A., 2016. Effects of dietary supplementation of Lactobacillus rhamnosus or/and Lactococcus lactis on the growth, gut microbiota and immune responses of red sea bream, Pagrus major. Fish Shellf. Immunol., 49: 275-285. https:// doi.org/10.1016/j.fsi.2015.12.047

Desai, R.A., Links, G.M., Collins, A.S., Mansfield, S.G., Drew, D.M., Kessel, V.G.A. and Hill, E.J., 2012. Effects of plant-based diets on the distal gut microbiome of rainbow trout (Oncorhynchus mykiss). Aquaculture, 350-353:134-142. https:// doi.org/10.1016/j.aquaculture.2012.04.005

Hao, Y.T., Wu, S.G., Jakovlić, I., Zou, H., Li, W.X. and Wang, G.T., 2017. Impacts of diet on hindgut microbiota and short-chain fatty acids in grass carp (Ctenopharyngodon idellus). Aquacult. Res., 48: 5595-5605. https://doi.org/10.1111/are.13381

He, S., Wu, Z., Liu, Y., Wu, N., Tao, Y., Xu, L., Zhou, Z., Yao, B. and Ringo, E., 2013. Effects of dietary 60 $\mathrm{g} \mathrm{kg}^{-1}$ dried distiller's grains in least-cost practical diets on production and gut allochthonous bacterial composition of cage-cultured fish: comparison among fish species with different natural food habits. Aquacult. Nutr., 19: 765-772. https://doi. org/10.1111/anu.12023

Ingerslev, H.C., von Gersdorff, J.L., Strube, M.L., Larsen, N., Dalsgaard, I., Boye, M. and Madsen, L., 2014. The development of the gut microbiota in rainbow trout (Oncorhynchus mykiss) is affected by first feeding and diet type. Aquaculture, 424-425: 24-34. https://doi.org/10.1016/j. aquaculture.2013.12.032

Jiang, Y., Xie, C., Yang, G., Gong, X., Chen, X., Xu, L. and Bao, B., 2011. Cellulase-producing bacteria of Aeromonas are dominant and indigenous in the gut of Ctenopharyngodon idellus (Valenciennes). Aquacult. Res., 42: 499-505. https://doi. org/10.1111/j.1365-2109.2010.02645.x

Larsen, A.M., Mohammed, H.H., Arias, C.R., 2014. Characterization of the gut microbiota of three commercially valuable warmwater fish species. J. appl. Microbiol., 116: 1396-1404. https://doi. org/10.1111/jam.12475

Li, W. and Godzik, A., 2006. Cd-hit: a fast program for clustering and comparing large sets of protein or nucleotide sequences. Bioinformatics, 22: 16581659. https://doi.org/10.1093/bioinformatics/ btl158

Li, X., Yu, Y., Feng, W., Yan, Q. and Gong, Y., 2012. Host species as a strong determinant of the intestinal microbiota of fish larvae. J. Microbiol., 50: 29-37. https://doi.org/10.1007/s12275-012-1340-1
Li, J., Ni, J., Li, J., Wang, C., Li, X., Wu, S., Zhang, T., Yu, Y. and Yan, Q., 2014. Comparative study on gastrointestinal microbiota of eight fish species with different feeding habits. J. appl. Microbiol., 117: 1750-1760. https://doi.org/10.1111/jam.12663

Li, T., Long, M., Gatesoupe, F., Zhang, Q., Li, A. and Gong, X., 2015. Comparative analysis of the intestinal bacterial communities in different species of carp by pyrosequencing. Microb. Ecol., 69: 2536. https://doi.org/10.1007/s00248-014-0480-8

Li, Z., Xu, L., Liu, W., Liu, Y., Ringø, E., Du, Z. and Zhou, Z., 2015. Protein replacement in practical diets altered gut allochthonous bacteria of cultured cyprinid species with different food habits. Aquacult. Int., 23: 913-928. https://doi.org/10.1007/ s10499-014-9851-x

Li, H., Zhong, Q., Wirth, S., Wang, W., Hao, Y., Wu, S., Zou, H., Li, W. and Wang, G., 2015. Diversity of autochthonous bacterial communities in the intestinal mucosa of grass carp (Ctenopharyngodon idellus) (Valenciennes) determined by culturedependent and culture independent techniques. Aquacult. Res., 46: 2344-2359. https://doi. org/10.1111/are.12391

Li, X., Zhou, L., Yu, Y., Ni, J., Xu, W. and Yan, Q. 2017. Composition of gut microbiota in the Gibel carp (Carassius auratus gibelio) varies with host development. Microb. Ecol., 74: 239-249. https:// doi.org/10.1007/s00248-016-0924-4

Nam, L.H., Chang, Y.S., Hong, H.B. and Lee, Y.E., 2003. A novel catabolic activity of Pseudomonas veronii in biotransformation of pentachlorophenol. Appl. Microbiol. Biotechnol., 62: 284-290. https:// doi.org/10.1007/s00253-003-1255-1

Reveco, E.F., Øverland, M., Romarheim, H.O. and Mydland, T.L., 2014. Intestinal bacterial community structure differs between healthy and inflamed intestines in Atlantic salmon (Salmo salar L.). Aquaculture, 420-421: 262-269. https://doi. org/10.1016/j.aquaculture.2013.11.007

Reyon, D., Tsai, S.Q., Khayter, C., Foden, J.A., Sander, J.D. and Joung, J.K., 2012. FLASH assembly of TALENs enables high-throughput genome editing. Nat. Biotechnol., 30: 460-465. https://doi. org/10.1038/nbt.2170

Roeselers, G., Mittge, E.K., Stephens, W.Z., Parichy, D.M., Cavanaugh, C.M., Guillemin, K. and Rawls, J.F., 2011. Evidence for a core gut microbiota in the zebrafish. ISME J., 5: 1595-1608. https://doi. org/10.1038/ismej.2011.38

Sullam, K.E., Essinger, S.D., Lozupone, C.A., O’Connor, M.P., Rosen, G.L., Knight, R., Kilham, 
S.S. and Russell, J.A., 2012. Environmental and ecological factors that shape the gut bacterial communities of fish: a meta-analysis. Mol. Ecol., 21: 3363-3378. https://doi.org/10.1111/j.1365294X.2012.05552.x

Tsuchiya, C., Sakata, T. and Sugita, H., 2008. Novel ecological niche of Cetobacterium somerae, an anaerobic bacterium in the intestinal tracts of freshwater fish. Lett. appl. Microbiol., 46: 43-48.

van Kessel, M.A., Dutilh, B.E., Neveling, K., Kwint, M.P., Veltman, J.A., Flik, G., Jetten, M.S., Klaren, P.H. and Camp, H.J.O., 2011. Pyrosequencing of 16S rRNA gene amplicons to study the microbiota in the gastrointestinal tract of carp (Cyprinus carpio L.). $A M B$ Express, 1: 41. https://doi. org/10.1186/2191-0855-1-41

Wu, S., Wang, G., Angert, R.E., Wang, W., Li, W. and Zou, H., 2012. Composition, diversity, and origin of the bacterial community in grass carp intestine.
PLoS One, 7: e30440. https://doi.org/10.1371/ journal.pone.0030440

Wu, S.G., Tian, J.Y., Gatesoupe, F.J., Li, W.X., Zou, H., Yang, B.J. and Wang, G.T., 2013. Intestinal microbiota of gibel carp (Carassius auratus gibelio) and its origin as revealed by 454 pyrosequencing. World J. Microbiol. Biotechnol., 29: 1585-1595. https://doi.org/10.1007/s11274-013-1322-4

Ye, L., Amberg, J., Chapman, D., Gaikowski, M. and Liu, W.T., 2014. Fish gut microbiota analysis differentiates physiology and behavior of invasive Asian carp and indigenous American fish. ISME J., 8: 541-551. https://doi.org/10.1038/ismej.2013.181

Zhang, J., Xiong, F., Wang, G.T., Li, W.X., Li, M., Zou, H. and Wu, S.G., 2017. The influence of diet on the grass carp intestinal microbiota and bile acids. Aquacult. Res., 48: 4934-4944. https://doi. org/10.1111/are.13312 